Proyecciones Journal of Mathematics

Vol. 35, No 1, pp. 119-136, March 2016.

Universidad Católica del Norte

Antofagasta - Chile

\title{
Sum divisor cordial graphs
}

\author{
A. Lourdusamy \\ St. Xavier's College, India \\ and \\ F. Patrick \\ St. Xavier's College, India \\ Received : December 2015. Accepted : March 2016
}

\begin{abstract}
A sum divisor cordial labeling of a graph $G$ with vertex set $V$ is a bijection $f$ from $V(G)$ to $\{1,2, \cdots,|V(G)|\}$ such that an edge uv is assigned the label 1 if 2 divides $f(u)+f(v)$ and 0 otherwise, then the number of edges labeled with 0 and the number of edges labeled with 1 differ by at most 1 . A graph with a sum divisor cordial labeling is called a sum divisor cordial graph. In this paper, we prove that path, comb, star, complete bipartite, $K_{2}+m K_{1}$, bistar, jewel, crown, flower, gear, subdivision of the star, $K_{1,3} * K_{1, n}$ and square graph of $B_{n, n}$ are sum divisor cordial graphs.
\end{abstract}

Subjclass : $05 \mathrm{C} 78$.

Keywords : Sum divisor cordial, divisor cordial. 


\section{Introduction}

All graphs considered here are simple, finite, connected and undirected. We follow the basic notations and terminologies of graph theory as in [2]. A labeling of a graph is a map that carries the graph elements to the set of numbers, usually to the set of non-negative or positive integers. If the domain is the set of vertices the labeling is called vertex labeling. If the domain is the set of edges, then we speak about edge labeling. If the labels are assigned to both vertices and edges then the labeling is called total labeling. For a dynamic survey of various graph labeling, we refer to Gallian [1].

Definition 1.1. Let $G=(V(G), E(G))$ be a simple graph and $f: V(G) \rightarrow$ $\{1,2, \cdots,|V(G)|\}$ be a bijection. For each edge uv, assign the label 1 if either $f(u) \mid f(v)$ or $f(v) \mid f(u)$ and the label 0 otherwise. The function $f$ is called a divisor cordial labeling if $\left|e_{f}(0)-e_{f}(1)\right| \leq 1$. A graph which admits a divisor cordial labeling is called a divisor cordial graph.

Motivated by the concept of divisor cordial labeling, we introduce a new concept of divisor cordial labeling called sum divisor cordial labeling.

Definition 1.2. Let $G=(V(G), E(G))$ be a simple graph and $f: V(G) \rightarrow$ $\{1,2, \cdots,|V(G)|\}$ be a bijection. For each edge uv, assign the label 1 if $2 \mid(f(u)+f(v))$ and the label 0 otherwise. The function $f$ is called a sum divisor cordial labeling if $\left|e_{f}(0)-e_{f}(1)\right| \leq 1$. A graph which admits a sum divisor cordial labeling is called a sum divisor cordial graph.

Definition 1.3. The comb $P_{n} \odot K_{1}$ is the graph obtained from a path by attaching a pendant edge to each vertex of the path.

Definition 1.4. The bistar $B_{n, n}$ is the graph obtained by attaching the apex vertices of two copies of $K_{1, n}$ by an edge.

Definition 1.5. The complete bipartite graph is a simple bipartite graph such that every vertex in one of the bipartition subsets is joined to every vertex in the other bipartition subset. Any complete bipartite graph that has $m$ vertices in one of its subsets and $n$ vertices in other is denoted by $K_{n, m}$.

Definition 1.6. The join of two graphs $G_{1}$ and $G_{2}$ is denoted by $G_{1}+G_{2}$ and whose vertex set is $V\left(G_{1}+G_{2}\right)=V\left(G_{1}\right) \cup V\left(G_{2}\right)$ and edge set is $E\left(G_{1}+G_{2}\right)=E\left(G_{1}\right) \cup E\left(G_{2}\right) \cup\left\{u v: u \in V\left(G_{1}\right), v \in V\left(G_{2}\right)\right\}$. 
Definition 1.7. The jewel $J_{n}$ is the graph with vertex set $V\left(J_{n}\right)=\left\{u, v, x, y, u_{i}\right.$ : $1 \leq i \leq n\}$ and edge set $E\left(J_{n}\right)=\left\{u x, u y, x y, x v, y v, u u_{i}, v u_{i}: 1 \leq i \leq n\right\}$.

Definition 1.8. The crown $C_{n} \odot K_{1}$ is the graph obtained from a cycle by attaching a pendant edge to each vertex of the cycle.

Definition 1.9. The helm $H_{n}$ is the graph obtained from a wheel by attaching a pendant edge to each rim vertex. The flower $F l_{n}$ is the graph obtained from a helm by attaching each pendant vertex to the apex of the helm.

Definition 1.10. The gear $G_{n}$ is the graph obtained from a wheel by subdividing each of its rim edge.

Definition 1.11. $K_{1,3} * K_{1, n}$ is the graph obtained from $K_{1,3}$ by attaching root of a star $K_{1, n}$ at each pendant vertex of $K_{1,3}$.

Definition 1.12. For a simple connected graph $G$ the square of graph $G$ is denoted by $G^{2}$ and defined as the graph with the same vertex set as of $G$ and two vertices are adjacent in $G^{2}$ if they are at a distance 1 or 2 apart in $G$.

Definition 1.13. The subdivision of star $S\left(K_{1, n}\right)$ is the graph obtained from $K_{1, n}$ by attaching a pendant edge to each vertex of $K_{1, n}$ except root vertex.

\section{2. main results}

Theorem 2.1. The path $P_{n}$ is sum divisor cordial graph.

Proof. Let $P_{n}$ be a path with consecutive vertices $v_{1}, v_{2}, \cdots, v_{n}$. Then $P_{n}$ is of order $n$ and size $n-1$. Define $f: V\left(P_{n}\right) \rightarrow\{1,2, \cdots, n\}$ as follows:

Case 1: $n$ is odd

$$
f\left(v_{i}\right)= \begin{cases}i & \text { if } i \equiv 0,1(\bmod 4) \\ i+1 & \text { if } i \equiv 2(\bmod 4) \text { for } 1 \leq i \leq n \\ i-1 & \text { if } i \equiv 3(\bmod 4)\end{cases}
$$


Case 2: $n$ is even

$$
f\left(v_{i}\right)= \begin{cases}i & \text { if } i \equiv 1,2(\bmod 4) \\ i+1 & \text { if } i \equiv 3(\bmod 4) \text { for } 1 \leq i \leq n \\ i-1 & \text { if } i \equiv 0(\bmod 4)\end{cases}
$$

In both cases, the induced edge labels are

$$
f^{*}\left(v_{i} v_{i+1}\right)= \begin{cases}1 & \text { if } 2 \mid\left(f\left(v_{i}\right)+f\left(v_{i+1}\right)\right) \\ 0 & \text { otherwise }\end{cases}
$$

We observe that,

$$
\begin{aligned}
& e_{f}(0)= \begin{cases}\frac{n-1}{2} & \text { if } n \text { is odd } \\
\frac{n}{2} & \text { if } n \text { is even }\end{cases} \\
& e_{f}(1)= \begin{cases}\frac{n-1}{2} & \text { if } n \text { is odd } \\
\frac{n-2}{2} & \text { if } n \text { is even }\end{cases}
\end{aligned}
$$

Thus, $\left|e_{f}(0)-e_{f}(1)\right| \leq 1$.

Hence, the path $P_{n}$ is sum divisor cordial graph.

Example 2.2. A sum divisor cordial labeling of $P_{6}$ and $P_{7}$ is shown in Figure 2.1
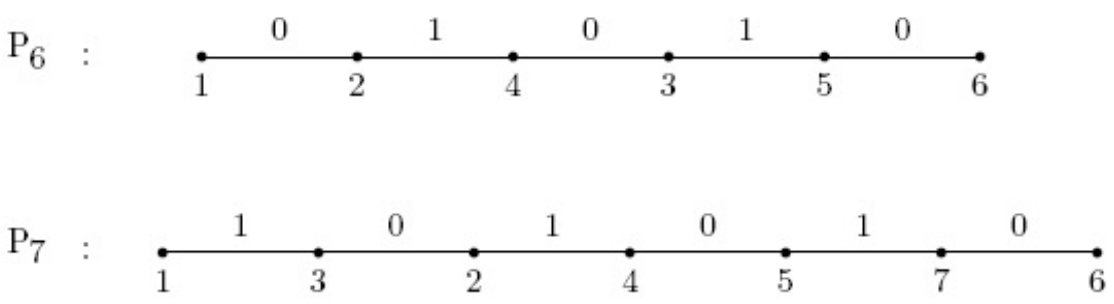

Figure 2.1

Theorem 2.3. The comb is sum divisor cordial graph.

Proof. Let $G$ be a comb obtained from the path $v_{1}, v_{2}, \cdots, v_{n}$ by joining a vertex $u_{i}$ to $v_{i}$ for each $i=1,2, \cdots, n$. Then $G$ is of order $2 n$ and size $2 n-1$. Define $f: V(G) \rightarrow\{1,2, \cdots, 2 n\}$ as follows: 


$$
\begin{gathered}
f\left(v_{i}\right)=2 i-1 ; 1 \leq i \leq n \\
f\left(u_{i}\right)=2 i ; 1 \leq i \leq n
\end{gathered}
$$

Then, the induced edge labels are

$$
\begin{gathered}
f^{*}\left(v_{i} v_{i+1}\right)=1 ; 1 \leq i \leq n-1 \\
f^{*}\left(v_{i} u_{i}\right)=0 ; 1 \leq i \leq n
\end{gathered}
$$

We observe that, $e_{f}(0)=n$ and $e_{f}(1)=n-1$. Thus, $\left|e_{f}(0)-e_{f}(1)\right| \leq 1$.

Hence, the comb is sum divisor cordial graph.

Example 2.4. A sum divisor cordial labeling of comb is shown in Figure 2.2

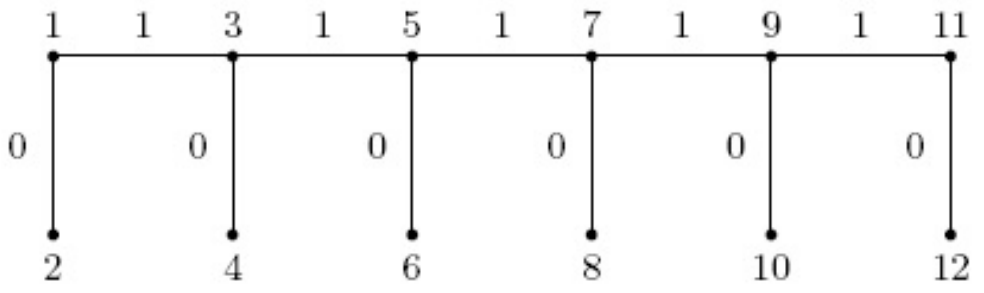

Figure 2.2

Theorem 2.5. The star $K_{1, n}$ is sum divisor cordial graph.

Proof. Let $\left(V_{1}, V_{2}\right)$ be the bipartition of $K_{1, n}$ with $V_{1}=\{u\}$ and $V_{2}=\left\{u_{1}, u_{2}, \cdots, u_{n}\right\}$. Let $E\left(K_{1, n}\right)=\left\{u u_{i}: 1 \leq i \leq n\right\}$. Then $K_{1, n}$ is of order $n+1$ and size $n$. Define $f: V\left(K_{1, n}\right) \rightarrow\{1,2, \cdots, n+1\}$ as follows:

$$
\begin{gathered}
f(u)=1 ; \\
f\left(u_{i}\right)=i+1 ; 1 \leq i \leq n
\end{gathered}
$$

Then, the induced edge labels are

$$
f^{*}\left(u u_{2 i-1}\right)=0 ; 1 \leq i \leq\left\lceil\frac{n}{2}\right\rceil
$$




$$
f^{*}\left(u u_{2 i}\right)=1 ; 1 \leq i \leq\left\lfloor\frac{n}{2}\right\rfloor
$$

We observe that,

$$
\begin{aligned}
& e_{f}(0)= \begin{cases}\frac{n+1}{2} & \text { if } n \text { is odd } \\
\frac{n}{2} & \text { if } n \text { is even }\end{cases} \\
& e_{f}(1)= \begin{cases}\frac{n-1}{2} & \text { if } n \text { is odd } \\
\frac{n}{2} & \text { if } n \text { is even }\end{cases}
\end{aligned}
$$

Thus, $\left|e_{f}(0)-e_{f}(1)\right| \leq 1$.

Hence, the star $K_{1, n}$ is sum divisor cordial graph.

Example 2.6. A sum divisor cordial labeling of $K_{1,5}$ is shown in Figure 2.3 .

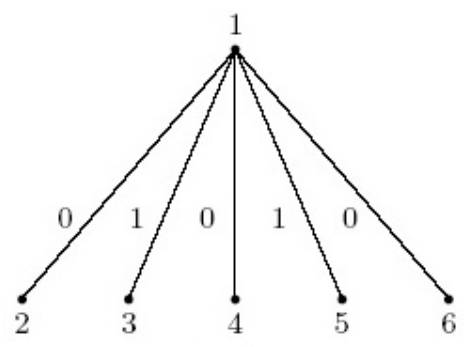

Figure 2.3

Theorem 2.7. The graph $K_{2, n}$ is sum divisor cordial graph.

Proof. Let $\left(V_{1}, V_{2}\right)$ be the bipartition of $K_{2, n}$ with $V_{1}=\{u, v\}$ and $V_{2}=\left\{v_{1}, v_{2}, \cdots, v_{n}\right\}$. Let $E\left(K_{2, n}\right)=\left\{u v_{i}, v v_{i}: 1 \leq i \leq n\right\}$. Then $K_{2, n}$ is of order $n+2$ and size $2 n$. Define $f: V\left(K_{2, n}\right) \rightarrow\{1,2, \cdots, n+2\}$ as follows:

$$
\begin{aligned}
& f(u)=1 \\
& f(v)=2 ; \\
& f\left(v_{i}\right)=i+2 ; 1 \leq i \leq n
\end{aligned}
$$

Then, the induced edge labels are

$$
\begin{aligned}
& f^{*}\left(u v_{2 i-1}\right)=1 ; 1 \leq i \leq\left\lceil\frac{n}{2}\right\rceil \\
& f^{*}\left(u v_{2 i}\right)=0 ; 1 \leq i \leq\left\lfloor\frac{n}{2}\right\rfloor \\
& f^{*}\left(v v_{2 i-1}\right)=0 ; 1 \leq i \leq\left\lceil\frac{n}{2}\right\rceil
\end{aligned}
$$




$$
f^{*}\left(v v_{2 i}\right)=1 ; 1 \leq i \leq\left\lfloor\frac{n}{2}\right\rfloor
$$

We observe that, $e_{f}(0)=e_{f}(1)=n$.

Thus, $\left|e_{f}(0)-e_{f}(1)\right| \leq 1$.

Hence, $K_{2, n}$ is sum divisor cordial graph.

Example 2.8. A sum divisor cordial labeling of $K_{2,5}$ is shown in Figure 2.4 .

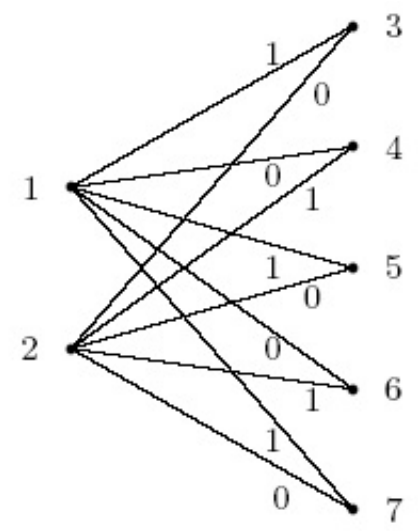

Figure 2.4

Theorem 2.9. The graph $K_{2}+m K_{1}$ is sum divisor cordial graph.

Proof. Let $G=K_{2}+m K_{1}$. Let $V(G)=\left\{u, v, w_{1}, w_{2}, \cdots, w_{m}\right\}$ and $E(G)=\left\{u v, u w_{i}, v w_{i}: 1 \leq i \leq m\right\}$. Then $G$ is of order $m+2$ and size $2 m+1$. Define $f: V(G) \rightarrow\{1,2, \cdots, m+2\}$ as follows:

$$
\begin{aligned}
& f(u)=1 ; \\
& f(v)=2 ; \\
& f\left(w_{i}\right)=i+2 ; 1 \leq i \leq m .
\end{aligned}
$$

Then, the induced edge labels are

$$
\begin{aligned}
& f^{*}(u v)=0 ; \\
& f^{*}\left(u w_{2 i-1}\right)=1 ; 1 \leq i \leq\left\lceil\frac{m}{2}\right\rceil \\
& f^{*}\left(u w_{2 i}\right)=0 ; 1 \leq i \leq\left\lfloor\frac{m}{2}\right\rfloor \\
& f^{*}\left(v w_{2 i-1}\right)=0 ; 1 \leq i \leq\left\lceil\frac{m}{2}\right\rceil \\
& f^{*}\left(v w_{2 i}\right)=1 ; 1 \leq i \leq\left\lfloor\frac{m}{2}\right\rfloor
\end{aligned}
$$


We observe that, $e_{f}(0)=m+1$ and $e_{f}(1)=m$.

Thus, $\left|e_{f}(0)-e_{f}(1)\right| \leq 1$.

Hence, $K_{2}+m K_{1}$ is sum divisor cordial graph.

Example 2.10. A sum divisor cordial labeling of $K_{2}+5 K_{1}$ is shown in Figure 2.5.

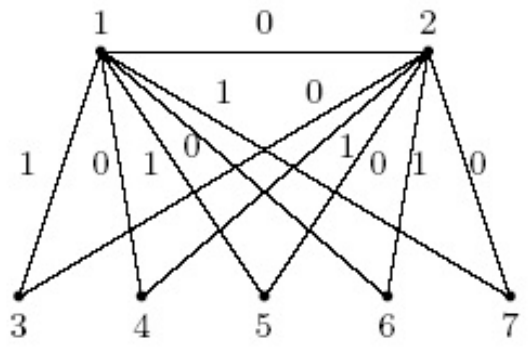

Figure 2.5

Theorem 2.11. The bistar $B_{n, n}$ is sum divisor cordial graph.

Proof. Let $G=B_{n, n}$. Let $V(G)=\left\{u, v, u_{i}, v_{i}: 1 \leq i \leq n\right\}$ and $E(G)=\left\{u v, v v_{i}, u u_{i}: 1 \leq i \leq n\right\}$. Then $G$ is of order $2 n+2$ and size $2 n+1$. Define $f: V(G) \rightarrow\{1,2, \cdots, 2 n+2\}$ as follows:

$$
\begin{aligned}
& f(u)=1 ; \\
& f(v)=2 ; \\
& f\left(u_{2 i-1}\right)=4 i-1 ; 1 \leq i \leq\left\lceil\frac{n}{2}\right\rceil \\
& f\left(u_{2 i}\right)=4 i+2 ; 1 \leq i \leq\left\lfloor\frac{n}{2}\right\rfloor \\
& f\left(v_{2 i-1}\right)=4 i ; 1 \leq i \leq\left\lceil\frac{n}{2}\right\rceil \\
& f\left(v_{2 i}\right)=4 i+1 ; 1 \leq i \leq\left\lfloor\frac{n}{2}\right\rfloor
\end{aligned}
$$

Then, the induced edge labels are

$$
\begin{aligned}
& f^{*}(u v)=0 ; \\
& f^{*}\left(u u_{2 i-1}\right)=1 ; 1 \leq i \leq\left\lceil\frac{n}{2}\right\rceil \\
& f^{*}\left(u u_{2 i}\right)=0 ; 1 \leq i \leq\left\lfloor\frac{n}{2}\right\rfloor \\
& f^{*}\left(v v_{2 i-1}\right)=1 ; 1 \leq i \leq\left\lceil\frac{n}{2}\right\rceil \\
& f^{*}\left(v v_{2 i}\right)=0 ; 1 \leq i \leq\left\lfloor\frac{n}{2}\right\rfloor
\end{aligned}
$$

We observe that,

$$
e_{f}(0)= \begin{cases}n & \text { if } n \text { is odd } \\ n+1 & \text { if } n \text { is even }\end{cases}
$$




$$
e_{f}(1)= \begin{cases}n+1 & \text { if } n \text { is odd } \\ n & \text { if } n \text { is even }\end{cases}
$$

Thus, $\left|e_{f}(0)-e_{f}(1)\right| \leq 1$.

Hence, the bistar $B_{n, n}$ is sum divisor cordial graph.

Example 2.12. A sum divisor cordial labeling of $B_{5,5}$ is shown in Figure 2.6 .

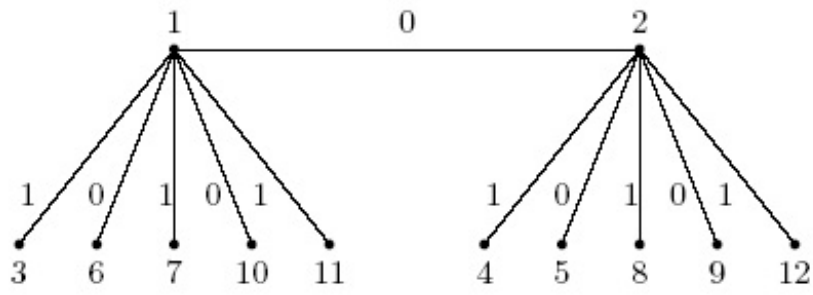

Figure 2.6

Theorem 2.13. The flower $F l_{n}$ is sum divisor cordial graph.

Proof. Let $G=F l_{n}$. Let $V(G)=\left\{v, v_{i}, u_{i}: 1 \leq i \leq n\right\}$ and $E(G)=\left\{v v_{i}, v_{i} u_{i}, v u_{i}: 1 \leq i \leq n ; v_{n} v_{1} ; v_{i} v_{i+1}: 1 \leq i \leq n-1\right\}$. Then $G$ is of order $2 n+1$ and size $4 n$. Define $f: V(G) \rightarrow\{1,2, \cdots, 2 n+1\}$ as follows:

$$
\begin{gathered}
f(v)=1 ; \\
f\left(v_{i}\right)=2 i ; 1 \leq i \leq n \\
f\left(u_{i}\right)=2 i+1 ; 1 \leq i \leq n
\end{gathered}
$$

Then, the induced edge labels are

$$
\begin{aligned}
& f^{*}\left(v v_{i}\right)=0 ; 1 \leq i \leq n \\
& f^{*}\left(v u_{i}\right)=1 ; 1 \leq i \leq n \\
& f^{*}\left(v_{i} u_{i}\right)=0 ; 1 \leq i \leq n \\
& f^{*}\left(v_{i} v_{i+1}\right)=1 ; 1 \leq i \leq n-1 \\
& f^{*}\left(v_{n} v_{1}\right)=1 ;
\end{aligned}
$$


We observe that, $e_{f}(0)=e_{f}(1)=2 n$.

Thus, $\left|e_{f}(0)-e_{f}(1)\right| \leq 1$

Hence, $F l_{n}$ is sum divisor cordial graph.

Example 2.14. A sum divisor cordial labeling of $\mathrm{Fl}_{4}$ is shown in Figure 2.7 .

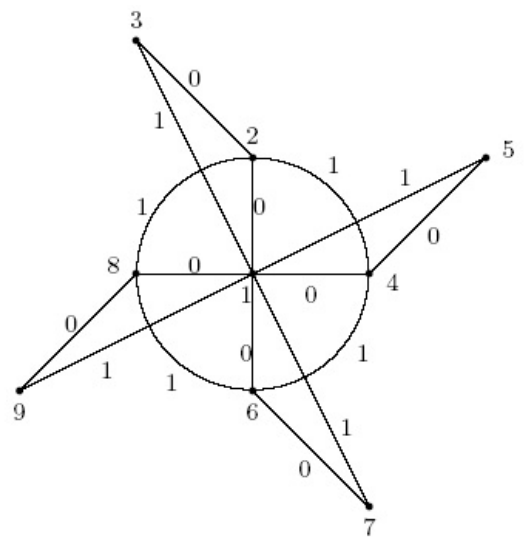

Figure 2.7

Theorem 2.15. The jewel $J_{n}$ is sum divisor cordial graph.

Proof. Let $G=J_{n}$. Let $V(G)=\left\{u, v, x, y, u_{i}: 1 \leq i \leq n\right\}$ and $E(G)=\left\{u x, u y, x y, x v, y v, u u_{i}, v u_{i}: 1 \leq i \leq n\right\}$. Then $G$ is of order $n+4$ and size $2 n+5$. Define $f: V(G) \rightarrow\{1,2, \cdots, n+4\}$ as follows:

$$
\begin{aligned}
& f(u)=1 ; \\
& f(v)=2 ; \\
& f(x)=3 ; \\
& f(y)=4 ; \\
& f\left(u_{i}\right)=i+4 ; 1 \leq i \leq n .
\end{aligned}
$$

Then, the induced edge labels are

$$
\begin{aligned}
& f^{*}(u x)=1 ; \\
& f^{*}(u y)=0 ; \\
& f^{*}(x y)=0 ; \\
& f^{*}(v x)=0 ; \\
& f^{*}(v y)=1 ; \\
& f^{*}\left(u u_{2 i-1}\right)=1 ; 1 \leq i \leq\left\lceil\frac{n}{2}\right\rceil \\
& f^{*}\left(u u_{2 i}\right)=0 ; 1 \leq i \leq\left\lfloor\frac{n}{2}\right\rfloor \\
& f^{*}\left(v u_{2 i-1}\right)=0 ; 1 \leq i \leq\left\lceil\frac{n}{2}\right\rceil
\end{aligned}
$$




$$
f^{*}\left(v u_{2 i}\right)=1 ; 1 \leq i \leq\left\lfloor\frac{n}{2}\right\rfloor
$$

We observe that, $e_{f}(0)=n+3$ and $e_{f}(1)=n+2$.

Thus, $\left|e_{f}(0)-e_{f}(1)\right| \leq 1$.

Hence, $J_{n}$ is sum divisor cordial graph.

Example 2.16. A sum divisor cordial labeling of $J_{4}$ is shown in Figure 2.8 .

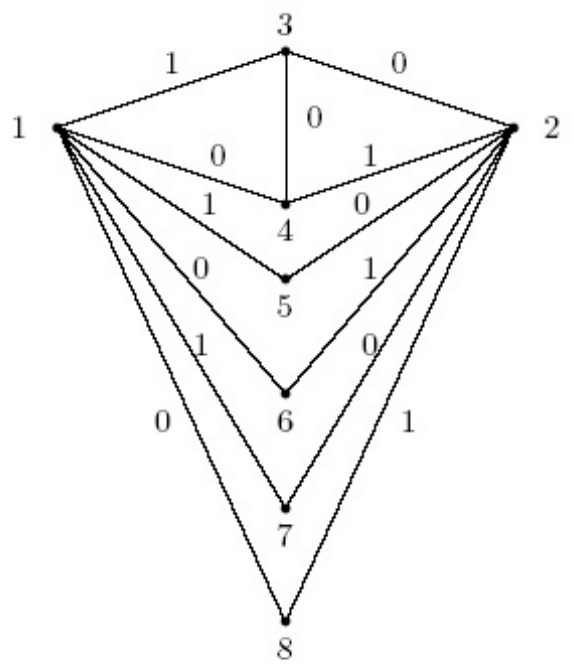

Figure 2.8

Theorem 2.17. The crown $C_{n} \odot K_{1}$ is sum divisor cordial graph.

Proof. Let $G=C_{n} \odot K_{1}$. Let $V(G)=\left\{u_{i}, v_{i}: 1 \leq i \leq n\right\}$ and $E(G)=\left\{u_{i} u_{i+1}: 1 \leq i \leq n-1 ; u_{n} u_{1}, u_{i} v_{i}: 1 \leq i \leq n\right\}$. Then $G$ is of order $2 n$ and size $2 n$. Define $f: V(G) \rightarrow\{1,2, \cdots, 2 n\}$ as follows:

$$
\begin{aligned}
& f\left(u_{i}\right)=2 i ; 1 \leq i \leq n \\
& f\left(v_{i}\right)=2 i-1 ; 1 \leq i \leq n
\end{aligned}
$$

Then, the induced edge labels are

$$
\begin{aligned}
& f^{*}\left(u_{i} u_{i+1}\right)=1 ; 1 \leq i \leq n-1 \\
& f^{*}\left(u_{n} u_{1}\right)=1 ; \\
& f^{*}\left(u_{i} v_{i}\right)=0 ; 1 \leq i \leq n
\end{aligned}
$$

We observe that, $e_{f}(0)=e_{f}(1)=n$.

Thus, $\left|e_{f}(0)-e_{f}(1)\right| \leq 1$.

Hence, $C_{n} \odot K_{1}$ is sum divisor cordial graph. 
Example 2.18. A sum divisor cordial labeling of $C_{4} \odot K_{1}$ is shown in Figure 2.9.

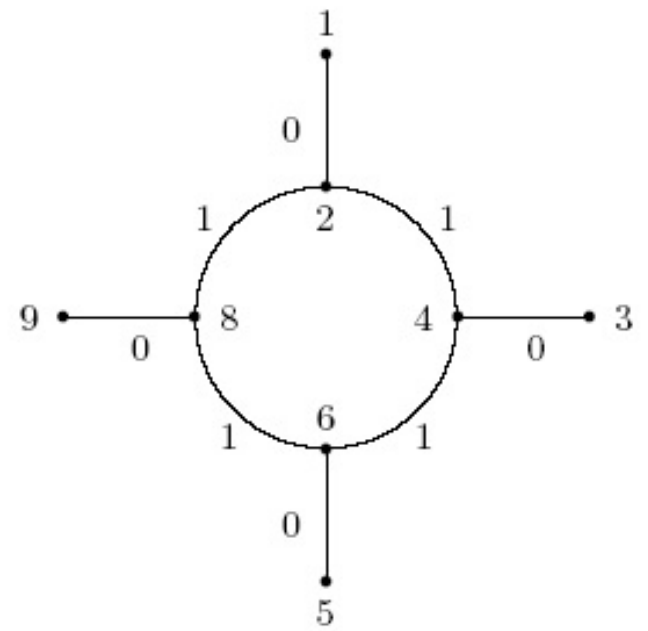

Figure 2.9

Theorem 2.19. The gear $G_{n}$ is sum divisor cordial graph.

Proof. Let $G=G_{n}$. Let $V(G)=\left\{v, u_{i}, v_{i}: 1 \leq i \leq n\right\}$ and $E(G)=\left\{v v_{i}, v_{i} u_{i}: 1 \leq i \leq n ; u_{i} v_{i+1}: 1 \leq i \leq n-1 ; u_{n} v_{1}\right\}$. Then $G$ is of order $2 n+1$ and size $3 n$. Define $f: V(G) \rightarrow\{1,2, \cdots, 2 n+1\}$ as follows:

$$
\begin{aligned}
& f(v)=1 ; \\
& f\left(v_{2 i-1}\right)=4 i-1 ; 1 \leq i \leq\left\lceil\frac{n}{2}\right\rceil \\
& f\left(v_{2 i}\right)=4 i ; 1 \leq i \leq\left\lfloor\frac{n}{2}\right\rfloor \\
& f\left(u_{2 i-1}\right)=4 i-2 ; 1 \leq i \leq\left\lceil\frac{n}{2}\right\rceil \\
& f\left(u_{2 i}\right)=4 i+1 ; 1 \leq i \leq\left\lfloor\frac{n}{2}\right\rfloor
\end{aligned}
$$

Then, the induced edge labels are

$$
\begin{aligned}
& f^{*}\left(v v_{2 i-1}\right)=1 ; 1 \leq i \leq\left\lceil\frac{n}{2}\right\rceil \\
& f^{*}\left(v v_{2 i}\right)=0 ; 1 \leq i \leq\left\lfloor\frac{n}{2}\right\rfloor \\
& f^{*}\left(v_{i} u_{i}\right)=0 ; 1 \leq i \leq n \\
& f^{*}\left(u_{i} v_{i+1}\right)=1 ; 1 \leq i \leq n-1 \\
& f^{*}\left(u_{n} v_{1}\right)= \begin{cases}0 & \text { if } n \text { is odd } \\
1 & \text { if } n \text { is even }\end{cases}
\end{aligned}
$$


We observe that, $e_{f}(0)=\left\lceil\frac{3 n}{2}\right\rceil$ and $e_{f}(1)=\left\lfloor\frac{3 n}{2}\right\rfloor$ Thus, $\left|e_{f}(0)-e_{f}(1)\right| \leq 1$.

Hence, $G_{n}$ is sum divisor cordial graph.

Example 2.20. A sum divisor cordial labeling of $G_{8}$ is shown in Figure 2.10 .

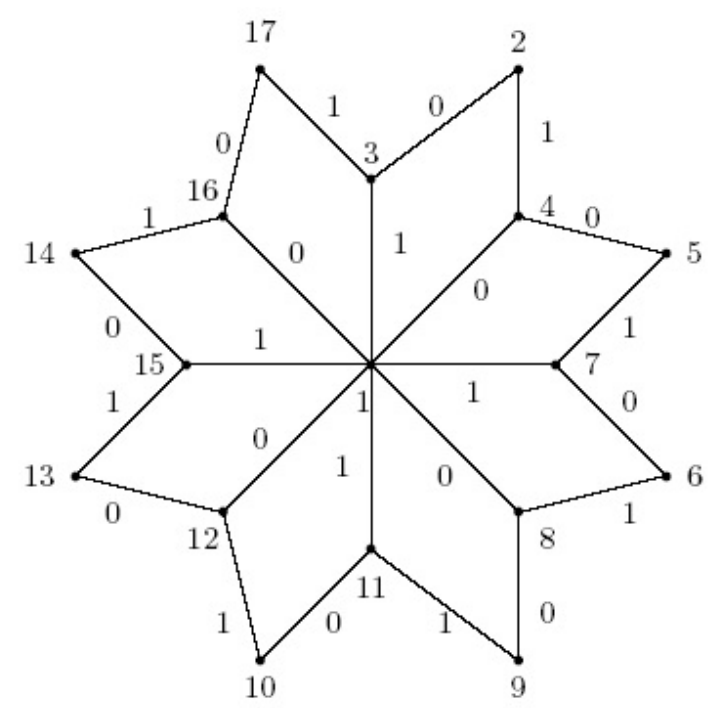

Figure 2.10

Theorem 2.21. The graph $K_{1,3} * K_{1, n}$ is sum divisor cordial graph.

Proof. Let $G=K_{1,3} * K_{1, n}$. Let $V(G)=\left\{x, u, v, w, u_{i}, v_{i}, w_{i}: 1 \leq i \leq\right.$ $n\}$ and $E(G)=\left\{x u, x v, x w, u u_{i}, v v_{i}, w w_{i}: 1 \leq i \leq n\right\}$. Then $G$ is of order $3 n+4$ and size $3 n+3$. Define $f: V(G) \rightarrow\{1,2, \cdots, 3 n+4\}$ as follows:

$$
\begin{aligned}
& f(u)=1 ; \\
& f(v)=2 ; \\
& f(w)=4 ; \\
& f(x)=3 ; \\
& f\left(u_{i}\right)=3 i+2 ; 1 \leq i \leq n \\
& f\left(v_{i}\right)=3 i+3 ; 1 \leq i \leq n \\
& f\left(w_{i}\right)=3 i+4 ; 1 \leq i \leq n
\end{aligned}
$$

Then, the induced edge labels are

$$
f^{*}(x u)=1
$$




$$
\begin{aligned}
& f^{*}(x v)=0 ; \\
& f^{*}(x w)=0 ; \\
& f^{*}\left(u u_{2 i-1}\right)=1 ; 1 \leq i \leq\left\lceil\frac{n}{2}\right\rceil \\
& f^{*}\left(u u_{2 i}\right)=0 ; 1 \leq i \leq\left\lfloor\frac{n}{2}\right\rfloor \\
& f^{*}\left(v v_{2 i-1}\right)=1 ; 1 \leq i \leq\left\lceil\frac{n}{2}\right\rceil \\
& f^{*}\left(v v_{2 i}\right)=0 ; 1 \leq i \leq\left\lfloor\frac{n}{2}\right\rfloor \\
& f^{*}\left(w w_{2 i-1}\right)=0 ; 1 \leq i \leq\left\lceil\frac{n}{2}\right\rceil \\
& f^{*}\left(w w_{2 i}\right)=1 ; 1 \leq i \leq\left\lfloor\frac{n}{2}\right\rfloor
\end{aligned}
$$

We observe that,

$$
\begin{aligned}
& e_{f}(0)= \begin{cases}\frac{3 n+3}{2} & \text { if } n \text { is odd } \\
\frac{3 n+4}{2} & \text { if } n \text { is even }\end{cases} \\
& e_{f}(1)= \begin{cases}\frac{3 n+3}{2} & \text { if } n \text { is odd } \\
\frac{3 n+2}{2} & \text { if } n \text { is even }\end{cases}
\end{aligned}
$$

Thus, $\left|e_{f}(0)-e_{f}(1)\right| \leq 1$.

Hence, $K_{1,3} * K_{1, n}$ is sum divisor cordial graph.

Example 2.22. A sum divisor cordial labeling of $K_{1,3} * K_{1,5}$ is shown in Figure 2.11.

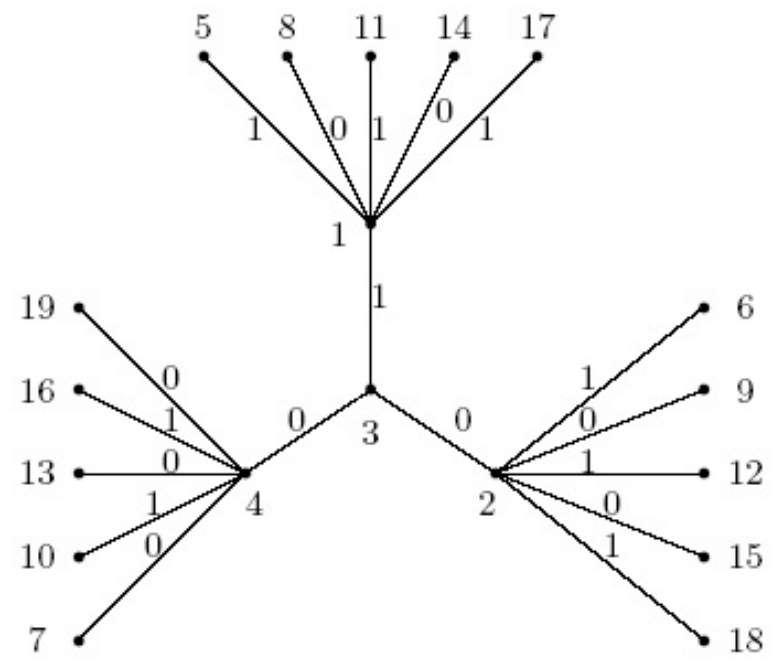

Figure 2.11

Theorem 2.23. The graph $B_{n, n}^{2}$ is sum divisor cordial graph. 
Proof. Let $G=B_{n, n}^{2}$. Let $V(G)=\left\{u, v, u_{i}, v_{i}: 1 \leq i \leq n\right\}$ and $E(G)=\left\{u v, v v_{i}, u u_{i}, u_{i} v, v_{i} u: 1 \leq i \leq n\right\}$. Then $G$ is of order $2 n+2$ and size $4 n+1$. Define $f: V(G) \rightarrow\{1,2, \cdots, 2 n+2\}$ as follows:

$$
\begin{aligned}
& f(u)=1 ; \\
& f(v)=2 ; \\
& f\left(u_{2 i-1}\right)=4 i-1 ; 1 \leq i \leq\left\lceil\frac{n}{2}\right\rceil \\
& f\left(u_{2 i}\right)=4 i+2 ; 1 \leq i \leq\left\lfloor\frac{n}{2}\right\rfloor \\
& f\left(v_{2 i-1}\right)=4 i ; 1 \leq i \leq\left\lceil\frac{n}{2}\right\rceil \\
& f\left(v_{2 i}\right)=4 i+1 ; 1 \leq i \leq\left\lfloor\frac{n}{2}\right\rfloor
\end{aligned}
$$

Then, the induced edge labels are

$$
\begin{aligned}
& f^{*}(u v)=0 ; \\
& f^{*}\left(u u_{2 i-1}\right)=1 ; 1 \leq i \leq\left\lceil\frac{n}{2}\right\rceil \\
& f^{*}\left(u u_{2 i}\right)=0 ; 1 \leq i \leq\left\lfloor\frac{n}{2}\right\rfloor \\
& f^{*}\left(v v_{2 i-1}\right)=1 ; 1 \leq i \leq\left\lceil\frac{n}{2}\right\rceil \\
& f^{*}\left(v v_{2 i}\right)=0 ; 1 \leq i \leq\left\lfloor\frac{n}{2}\right\rfloor \\
& f^{*}\left(v u_{2 i-1}\right)=0 ; 1 \leq i \leq\left\lceil\frac{n}{2}\right\rceil \\
& f^{*}\left(v u_{2 i}\right)=1 ; 1 \leq i \leq\left\lfloor\frac{n}{2}\right\rfloor \\
& f^{*}\left(u v_{2 i-1}\right)=0 ; 1 \leq i \leq\left\lceil\frac{n}{2}\right\rceil \\
& f^{*}\left(u v_{2 i}\right)=1 ; 1 \leq i \leq\left\lfloor\frac{n}{2}\right\rfloor
\end{aligned}
$$

We observe that, $e_{f}(1)=2 n$ and $e_{f}(0)=2 n+1$.

Thus, $\left|e_{f}(0)-e_{f}(1)\right| \leq 1$.

Hence, the graph $B_{n, n}^{2}$ is sum divisor cordial graph.

Example 2.24. A sum divisor cordial labeling of $B_{4,4}^{2}$ is shown in Figure 2.12 .

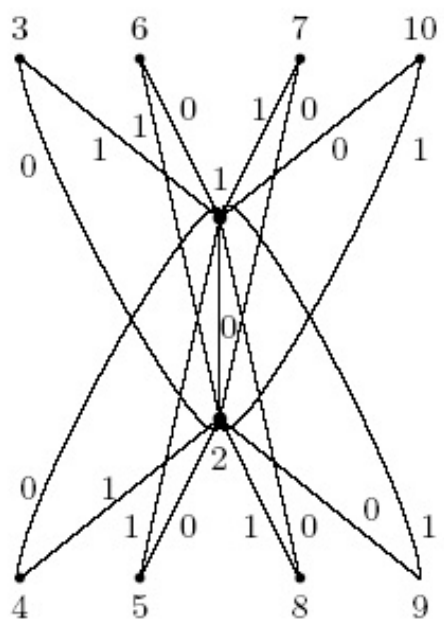

Figure 2.12 
Theorem 2.25. The graph $S\left(K_{1, n}\right)$ is sum divisor cordial graph.

Proof. Let $G=S\left(K_{1, n}\right)$. Let $V(G)=\left\{v, v_{i}, u_{i}: 1 \leq i \leq n\right\}$ and $E(G)=\left\{v v_{i}, v_{i} u_{i}: 1 \leq i \leq n\right\}$. Then $G$ is of order $2 n+1$ and size $2 n$. Define $f: V(G) \rightarrow\{1,2, \cdots, 2 n+1\}$ as follows:

$$
\begin{aligned}
& f(v)=1 ; \\
& f\left(v_{i}\right)=2 i+1 ; 1 \leq i \leq n \\
& f\left(u_{i}\right)=2 i ; 1 \leq i \leq n
\end{aligned}
$$

Then, the induced edge labels are

$$
\begin{aligned}
& f^{*}\left(v v_{i}\right)=1 ; 1 \leq i \leq n \\
& f^{*}\left(v_{i} u_{i}\right)=0 ; 1 \leq i \leq n
\end{aligned}
$$

We observe that, $e_{f}(0)=e_{f}(1)=n$.

Thus, $\left|e_{f}(0)-e_{f}(1)\right| \leq 1$.

Hence, $S\left(K_{1, n}\right)$ is sum divisor cordial graph.

Example 2.26. A sum divisor cordial labeling of $S\left(K_{1,5}\right)$ is shown in Figure 2.13 .

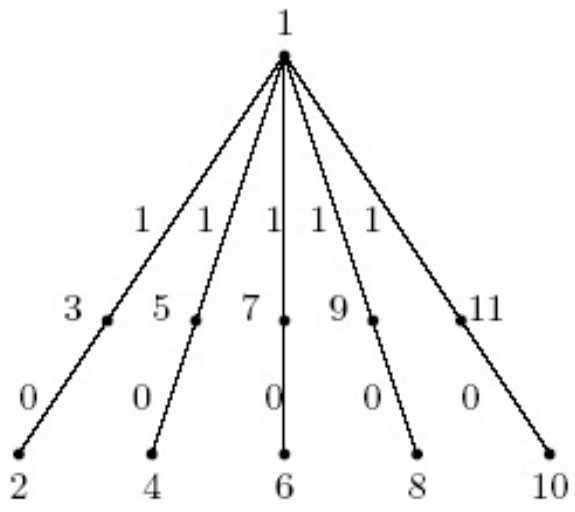

Figure 2.13

\section{3. conclusion}

All the graphs are not sum divisor cordial graphs. It is very interesting and challenging as well to investigate sum divisor cordial labeling for the 
graph or graph families which admit sum divisor cordial labeling. Here we have proved path, comb, star, complete bipartite, $K_{2}+m K_{1}$, bistar, jewel,

crown, flower, gear, subdivision of the star, $K_{1,3} * K_{1, n}$ and square graph of $B_{n, n}$ are sum divisor cordial graphs. In the subsequent paper, we will prove that total graph of the path, square graph of the path, shadow graph of the path and alternative triangular snake are sum divisor cordial graphs. Also, we will prove book, one point union of cycles, triangular ladder related graphs are sum divisor cordial graphs.

\section{References}

[1] J. A. Gallian, A Dyamic Survey of Graph Labeling, The Electronic J. Combin., 17 (2015) \#DS6.

[2] F. Harary, Graph Theory, Addison-wesley, Reading, Mass (1972).

[3] P. Lawrence Rozario Raj and R. Lawence Joseph Manoharan, Some Result on Divisor Cordial Labeling of Graphs, Int. J. Innocative Sci., 1 (10), pp. 226-231, (2014).

[4] P. Maya and T. Nicholas, Some New Families of Divisor Cordial Graph, Annals Pure Appl. Math., 5 (2), pp. 125-134, (2014).

[5] A. Nellai Murugan and G. Devakiruba, Cycle Related Divisor Cordial Graphs, Int. J. Math. Trends and Tech., 12 (1), pp. 34-43, (2014).

[6] A. Nellai Murugan, G. Devakiruba and S. Navanaeethakrishan, Star Attached Divisor Cordial Graphs, Int. J. Inno. Sci. Engineering and Tech., 1 (5), pp. 165-171, (2014).

[7] S. K. Vaidya and N. H. Shah, Some Star and Bistar Related Cordial Graphs, Annals Pure Appl. Math., 3 (1), pp. 67-77, (2013).

[8] S. K. Vaidya and N. H. Shah, Further Results on Divisor Cordial Labeling, Annals Pure Appl. Math., 4 (2), pp. 150-159, (2013).

[9] R. Varatharajan, S. Navanaeethakrishan and K. Nagarajan, Divisor Cordial Graphs, Int. J. Math. Combin., 4, pp. 15-25, (2011). 
A. Lourdusamy

Department of Mathematics,

St. Xavier's College,

Palayamkottai-627002,

Tamilnadu,

India

e-mail : lourdusamy15@gmail.com

and

F. Patrick

Department of Mathematics,

St. Xavier's College,

Palayamkottai-627002,

Tamilnadu,

India

e-mail : patrick881990@gmail.com 